Sabah Ahmed Hussein ${ }^{1}$, Khaled Mahmoud Kamel', Safy Zahid Kaddah', Emad Efat Abd El-Hamid², Marwa Moawad Shaban'

${ }^{1}$ Chest Department, Faculty of Medicine, Cairo University, Egypt

${ }^{2}$ Shebin Elkom Chest Hospital, Egypt

\title{
Role of ultrasonography in assessment of anatomic upper airway changes in patients with obstructive sleep apnea
}

\begin{abstract}
Instroduction: Obstructive sleep apnea is a common disorder, characterized by recurrent narrowing and closure of the upper airway accompanied by intermittent oxyhemoglobin desaturation and sympathetic activation. Ultrasound imaging of the airways has advantages of being safe, quick, repeatable, portable and widely available. Airway ultrasound can visualize and assess the mouth and tongue, oropharynx, hypopharynx, epiglottis, larynx, vocal cords, cricothyroid membrane, cricoid cartilage, trachea, and cervical esophagus.

Material and methods: This study assessed the role of ultrasonography in detecting the level and degree of obstruction of airway passages in patients with obstructive sleep apnea (OSA) and its relation to OSA severity. It included thirty-three patients diagnosed as OSA, and ten healthy subjects as a control group. All participants were $\geq 18$ years and were subjected to full medical history, Epworth sleepiness score (ESS), thorough clinical examination, complete overnight polysomnography and neck ultrasonography.

Results: Ultrasonography findings showed a statistically significant increase in lateral parapharyngeal wall thickness (LPWT) (P $<0.001)$ and a significant increase in distance between lingual arteries (DLA) ( $<0.01$ ) among OSA patients. Moreover, there was a significant statistical decrease in the retropalatal pharynx transverse diameter (RPD) $(P<0.05)$ in the OSA group compared to those without OSA. LPWT and DLA are parameters that can be used to predict the severity of OSA. Combination of LPWT and RPD can achieve a $100 \%$ sensitivity and specificity.

Conclusions: Ultrasound is more objective and convenient than the questionnaire because it doesn't require overnight time consumption. It is also more relevant than pulse oximetry for examining pharyngeal airspace. Also, this study demonstrated that submental ultrasonography is sufficiently sensitive for differentiating OSA severity.
\end{abstract}

Key words: obstructive sleep apnea, neck ultrasonography, upper airway anatomy

Adv Respir Med. 2020; 88: 548-557

\section{Introduction}

Obstructive sleep apnea is a common disorder, characterized by recurrent narrowing and closure of the upper airway accompanied by intermittent oxyhemoglobin desaturation and sympathetic activation. Diagnosis of OSA can be indicated by symptomatology and the presence of known risk factors such as increasing age, obesity and large neck circumference, although OSA can occur in individuals with none of these risk factors [1]. A number of tools and methods are available for the assessment of sleep health as self-reported questionnaire instruments. However, the gold standard for diagnosis of OSA is the overnight polysomnography [2].

Due to convenience, inexpensiveness, no radiation exposure and office-based procedure, an increasing number of physicians uses ultrasonography (US) to examine the neck. Several studies also demonstrate the role of US in detecting anatomic risk factors for OSA, including parapharyngeal pad and the tongue [3]. Airway ultrasound can visualize and assess the mouth

Address for correspondence: Sabah Ahmed Hussein, Chest Department, Faculty of Medicine, Cairo University, Egypt; e-mail: sabah.hussein@kasralainy.edu.eg DOI: 10.5603/ARM.a2020.0187

Received: 08.05.2020

Copyright (C) 2020 PTChP

ISSN 2451-4934 
and tongue, oropharynx, hypopharynx, epiglottis, larynx, vocal cords, cricothyroid membrane, cricoid cartilage, trachea and cervical esophagus. Neck ultrasound can measure coronal mid-tongue base thickness (CTBT), sagittal midtongue base thickness (STBT), retropalatal pharynx transverse diameter (RPD), distance between lingual arteries (DLA), lateral parapharyngeal wall thickness (LPWT). It can detect both the anatomic characteristics, the dynamic changes of pharyngeal airspace and discriminate OSA severity [4]. Different treatment options are now available for effective management of OSA. Positive airway pressure (PAP) provides pneumatic splinting of the upper airway and is effective in reducing apnea-hypopnea index (AHI). PAP may be delivered in continuous (CPAP), bi-level (BiPAP), or auto-titrating (APAP) modes [5].

This study investigated the role of ultrasonography in detection of the level and degree of obstruction of the airway passages in patients with OSA and its relation to OSA severity.

\section{Material and methods}

This is an analytical case/control study that was performed as a collaborative work between the sleep unit and chest ultrasonography unit, Chest Department, Faculty of Medicine, Cairo University, during the period between January 2017 and October 2018. The study included thirty-three patients diagnosed as OSA and ten healthy subjects as a control group. All patients met the following eligibility criteria: physician confirmed diagnosis of OSA, age $\geq 18$ years, and on a regular medication regimen for OSA. All persons with tracheostomy, evident underlying cause of any sleep disorders such as alcohol intake or endocrinal disorders such as hypothyroidism or acromegaly, patients with abnormalities in the soft palate or upper airway and those with underlying heart disease (e.g., organic valvular heart diseases, dysrhythmias, cardiac tamponade and pericardial effusion) were excluded from the study. The study was ethically approved by the institutional research ethics committee. Informed consent was obtained from all participants. All subjects underwent detailed clinical evaluation, including history taking, clinical examination, Epworth sleepiness score, Electrocardiogram, echocardiography. Complete overnight polysomnography was performed over a whole night (8-hour sleep). The study started at 10 P.M. until 6 A.M. using SOMNOscreen ${ }^{\mathrm{TM}}$ plus Polysomnography with digital IR-Videometry (Germany).
Data obtained included:

- Apnea: complete cessation of airflow breathing at the nostrils and mouth for at least 10 seconds or longer.

- Hypopnea: decrease in rate and depth of breathing by $50 \%$ for 10 seconds or longer.

- Apnea-hypopnea index (AHI): average number of apnea and hypopnea per hour of sleep. Persons with AHI $<5$ are not considered to have OSA. In contrast, AHI $\geq 5$ and $<15$, AHI $\geq 15$ and $<30$, and $\mathrm{AHI} \geq 30$ are classified as mild, moderate, and severe, respectively [6].

- Other measured variables are total sleep time, sleep efficiency, sleep stage percentage, sleep stage latency, arousals, respiratory disturbance index (RDI), snoring, body position, oxyhemoglobin saturation, limb movements and arrhythmias.

- The system records sleep stages by electroencephalography (EEG), electrooculography (EOG) and electromyography (EMG). EEG was used to monitor sleep stages and identify sleep latency and arousal. EOG was applied to monitor both horizontal and vertical eye movements to document the onset of REM and slow rolling movements accompanying the onset of sleep. EMG records atonia during REM or lack of atonia in REM-related parasomnia.

Neck ultrasonography was done using Hitachi EUB-7000 in the ultrasound unit, Chest Department, Kasr Al-Ainy Hospital. All cases were examined by B-mode and Doppler scan with curvilinear transducer $(2-5 \mathrm{MHz})$. Ultrasound examination was done on the day after overnight polysomnography. All sonographic measurements were made by the same well-trained operator in ultrasonography who was blinded to the polysomnographic data. The study subjects laid supine on the examination couch. The neck of the patient was slightly extended with the infraorbital meatal baseline (the line joining infraorbital margin and the ear tragus) perpendicular to the scanning table. Measurements obtained included:

- Retropalatal pharynx transverse diameter (RPD): ultrasonography scanning from the hyoid bone to the external auditory meatus at the level of oral pharynx, then the probe is tilted downward to locate the retropalatal pharynx which is defined as air column at the highest plane near the uvula. RPD is determined by the outer margin of the air column (RPD) (Figure 1A).

- Distance between lingual arteries (DLA): in the same previous ultrasonography scanning position, the lingual arteries were observed 

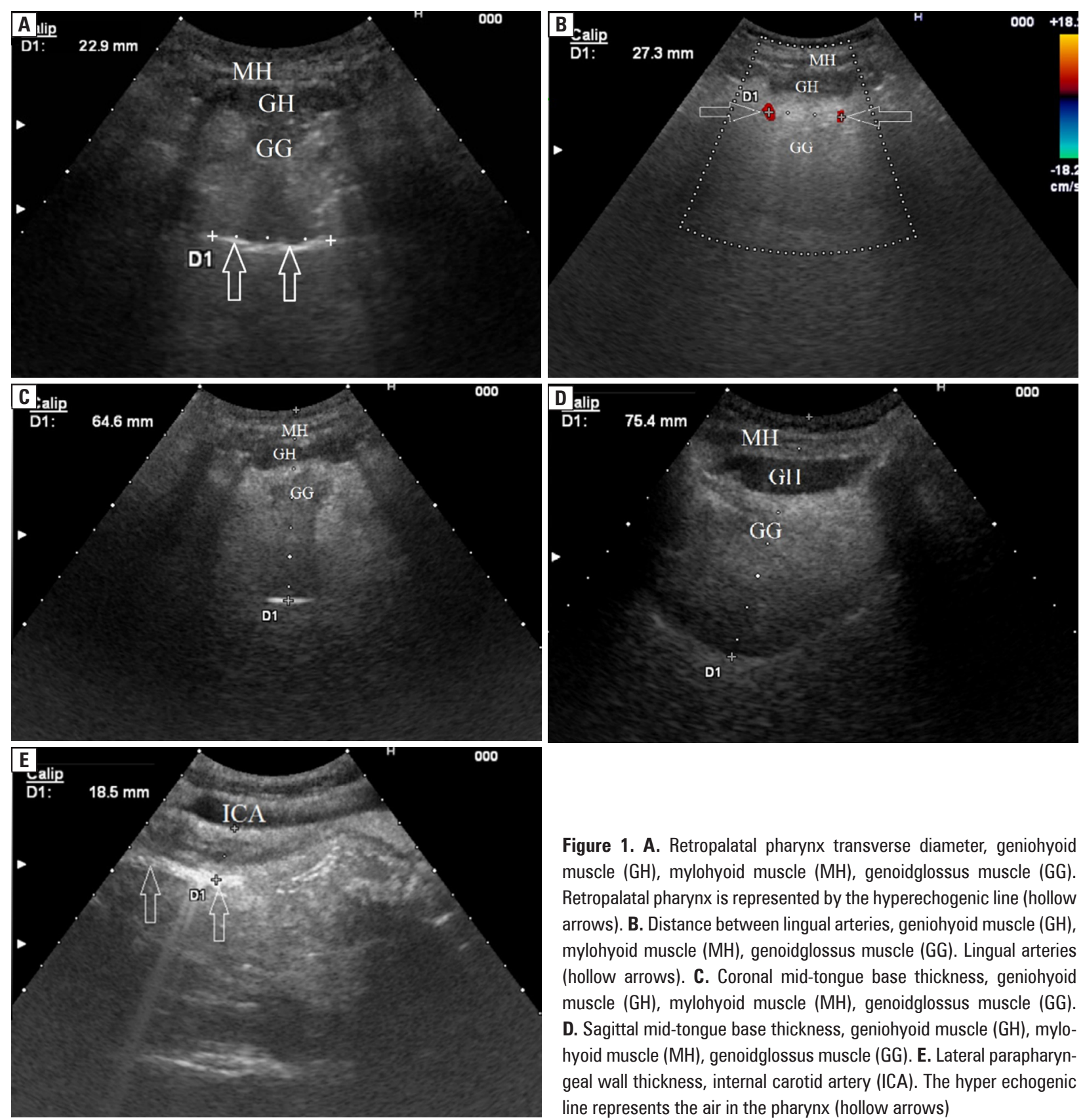

Figure 1. A. Retropalatal pharynx transverse diameter, geniohyoid muscle (GH), mylohyoid muscle (MH), genoidglossus muscle (GG). Retropalatal pharynx is represented by the hyperechogenic line (hollow arrows). B. Distance between lingual arteries, geniohyoid muscle (GH), mylohyoid muscle (MH), genoidglossus muscle (GG). Lingual arteries (hollow arrows). C. Coronal mid-tongue base thickness, geniohyoid muscle (GH), mylohyoid muscle (MH), genoidglossus muscle (GG). D. Sagittal mid-tongue base thickness, geniohyoid muscle (GH), mylohyoid muscle (MH), genoidglossus muscle (GG). E. Lateral parapharyngeal wall thickness, internal carotid artery (ICA). The hyper echogenic line represents the air in the pharynx (hollow arrows)

by power Doppler scan on both sides of the lower lateral border of the tongue base. The distance between lingual arteries (DLA) was measured (Figure 1B).

- Coronal mid-tongue base thickness (CTBT): in the same previous coronal ultrasonography scanning position, the mid-tongue base thickness (CTBT) was measured (Figure 1C).

- Sagittal mid-tongue base thickness (STBT): from the previous coronal ultrasonography scanning position the probe was rotated $90^{\circ}$ into the sagittal position, then the sagittal mid-tongue base thickness (STBT) was measured (Figure 1D).

- Lateral parapharyngeal wall thickness (LPWT): the oblique coronal plane of the parapharyngeal space was scanned with the transducer longitudinally placed on the lateral side of the neck, just underneath the lateral border of the occipital bone. The long axis of the ipsilateral internal carotid artery was identified with color application. The lateral wall of the pharynx appeared as an echogenic line on real-time ultrasound, whereas the lumen of the pharynx was completely obscured by gas shadowing. Vibration artifacts occasionally occurred when the subjects swallowed, which also helped to confirm the location of the 
pharynx. The distance between the internal carotid artery and the echogenic surface of the pharynx represented the LPW thickness in an oblique coronal plane. All the measurements were recorded on frozen images when the lateral wall of the pharynx moved farthest away from the transducer (Figure 1E).

\section{Statistical methods}

Data were statistically described in terms of mean \pm standard deviation ( $\pm \mathrm{SD}$ ), range, frequencies (number of cases) and/or percentages when appropriate. Correlation coefficient was used to find the strength of association between numerical variables - that is, anthropometric measurements, submental US measurements, and PSG parameters. Receiver operating characteristics (ROC) analysis was used to determine the optimal cutoff values of the individual anatomical US indices and individual anthropometric indices for predicting and screening for OSA using computer-generated randomization of the OSA patients with complete data, multivariate analysis of the patient group. Pearson correlation (r) was used to detect association between quantitative variables. Accuracy was represented using the terms sensitivity, specificity, + ve predictive value, -ve predictive value, and overall accuracy. Multivariate logistic regression analysis models were used to test for the preferential effect of the independent variable(s) on the occurrence of OSA. Probability (P-value) is considered significant if $<0.05$ level. All statistical calculations were performed manually as well as using computer program SPSS (Statistical Package for the Social Science; SPSS Inc., Chicago, IL, USA) version 22 for Microsoft Windows.

\section{Results}

This study comprised two groups. The group 1 included OSA patients $(\mathrm{n}=33)$ : 15 males (45.5\%) and 18 females (54.5\%), their age range was 39-75 years with mean \pm SD of $57.76 \pm$ 7.84 years, their body mass index (BMI) ranged from 28.10 to $66.10 \mathrm{~kg} / \mathrm{m}^{2}$ with mean \pm SD of $43.30 \pm 10.11 \mathrm{~kg} / \mathrm{m}^{2}$. They were subdivided according to polysomnography results into mild OSA $(5 \leq$ AHI $<15)$ patients $(9 / 33,27.2 \%)$, Moderate OSA $(15 \leq$ AHI $<30)$ patients $(12 / 33,36.4 \%)$ and Severe OSA $(A H I \geq 30)$ patients $(12 / 33,36.4 \%)$. The group 2 included 10 healthy individuals as a control group that comprised 5 males (50\%) and 5 females (50\%), their age range was 35-58 years with mean \pm SD of $48.00 \pm 7.85$ years, their BMI range was $25-45.80 \mathrm{~kg} / \mathrm{m}^{2}$ with mean \pm SD of $32.08 \pm 6.67 \mathrm{~kg} / \mathrm{m}^{2}$. The most common sleep-related symptoms among OSA patients were excessive daytime sleepiness (EDS) 28/33 (84.8\%), nocturia $27 / 33$ (81.8\%), snoring 26/33 (78.8\%) and morning headache 25/33 (75.8\%), and the difference between the cases and controls was statistically significant. There was a statistically significant difference between OSA cases and the controls regarding mean age $(57.76 \pm 7.84$ vs $48.0 \pm 7.85 ; \mathrm{P}<$ 0.01), $\mathrm{BMI}$ (mean $\pm \mathrm{SD}=43.30 \pm 10.11$ vs $32.08 \pm$ 6.67; $\mathrm{P}<0.001$ ), neck circumference (mean $\pm \mathrm{SD}$ $=43.24 \pm 2.69$ vs $38.20 \pm 1.23 ; \mathrm{P}<0.001)$ and ESS (mean \pm SD $=16.15 \pm 3.12$ vs $3.50 \pm 2.84$; $\mathrm{P}<0.001)$. No statistically significant difference between the cases and controls regarding diabetes mellitus and hypertension was found. But the difference in incidence of ischemic heart disease between OSA cases $(19 / 33,42.4 \%)$ and the controls $(1 / 10,10 \%)$ was statistically significant $(P<0.05)$.

Polysomnography and neck ultrasonography data of the studied groups was presented in Table 1, and it revealed a statistically significant difference between OSA cases and the controls regarding $\mathrm{A}+\mathrm{H} / \mathrm{h}$ in supine position, respiratory-related leg movements (Resp-LMs) index, minimal and average oxygen saturation (\%), $\mathrm{SpO}_{2}$ Time $<90 \%$, oxygen desaturation index, apnea index, hypopnea index, AHI and arrhythmia index. In regard to ultrasonography findings, anatomical parameters showed a statistically significant increase in LPWT $(\mathrm{P}<0.001)$ and DLA $(\mathrm{P}<0.01)$ among OSA patients in comparison to the controls. Moreover, there was a significant decrease or shortening in the RPD $(\mathrm{P}<0.05)$ in OSA patients than among the controls. There was a significant positive correlation between LPWT and neck circumference [correlation coefficient $(\mathrm{r}$ ) $=0.571, \mathrm{P}<0.001)$ and BMI $(\mathrm{r}=0.328, \mathrm{P}<0.05)$ and a significant positive correlation between DLA and neck circumference $(\mathrm{r}=0.384, \mathrm{P}<$ 0.05). In addition, there was a significant negative correlation between RPD in all the studied groups and neck circumference $(\mathrm{r}=-0.407, \mathrm{P}<0.01)$.

The correlation between neck ultrasonography findings and polysomnography parameters among all studied groups showed that LPWT is negatively correlated with baseline oxygen saturation $(\mathrm{r}=-0.320, \mathrm{P}<0.05)$ and average oxygen saturation $(\mathrm{r}=-0.483, \mathrm{P}<0.001)$, while it was positively correlated with $\mathrm{SpO}_{2}$ time $<90 \%(\mathrm{r}=$ $0.445, \mathrm{P}<0.01)$, oxygen desaturation index $(\mathrm{r}=$ 0.545, $\mathrm{P}<0.001)$, apnea index $(\mathrm{r}=0.393, \mathrm{P}<$ 0.01), hypopnea index ( $\mathrm{r}=0.550, \mathrm{P}<0.001)$, AHI $(\mathrm{r}=0.586, \mathrm{P}<0.001), \mathrm{A}+\mathrm{H} / \mathrm{h}$ in supine position 
Table 1. Polysomnography and ultrasonography data of the studied groups

\begin{tabular}{|c|c|c|c|}
\hline & \multicolumn{2}{|c|}{ The studied groups } & \multirow[t]{2}{*}{$P$ value } \\
\hline & OSA patients ( $N=33$ ) & Control $(\mathrm{N}=10)$ & \\
\hline \multicolumn{4}{|l|}{ Polysomnography data } \\
\hline Total sleep time (TST) [min] (mean \pm SD) & $292.85 \pm 90.42$ & $345.70 \pm 59.07$ & $>0.05$ \\
\hline Total sleep period (SPT) [min] mean \pm SD & $339.21 \pm 78.00$ & $377.50 \pm 39.51$ & $>0.05$ \\
\hline Sleep efficiency (mean \pm SD) & $85.63 \pm 20.20$ & $84.7 \pm 94.45$ & $>0.05$ \\
\hline Wake index (mean \pm SD) & $4.92 \pm 5.36$ & $7.08 \pm 8.55$ & $>0.05$ \\
\hline Flow limitation index $\%$ total (mean \pm SD) & $3.01 \pm 8.29$ & $13.67 \pm 12.32$ & $>0.05$ \\
\hline$\%$ of sleep in supine position (mean \pm SD) & $75.95 \pm 28.86$ & $81.48 \pm 22.70$ & $>0.05$ \\
\hline $\mathrm{A}+\mathrm{H} / \mathrm{h}$ in supine position (mean $\pm \mathrm{SD}$ ) & $28.00 \pm 21.98$ & $1.49 \pm 1.54$ & $<0.001$ \\
\hline Isolated LMs index (mean \pm SD) & $16.15 \pm 16.74$ & $16.35 \pm 13.18$ & $>0.05$ \\
\hline Resp-LMs index (mean \pm SD) & $29.94 \pm 39.80$ & $0.40 \pm 0.55$ & $<0.001$ \\
\hline Minimal oxygen saturation [\%] (mean \pm SD) & $68.09 \pm 12.63$ & $83.40 \pm 10.12$ & $<0.001$ \\
\hline Baseline oxygen saturation [\%] (mean \pm SD) & $90.58 \pm 5.88$ & $92.90 \pm 4.28$ & $>0.05$ \\
\hline Average oxygen saturation [\%] (mean $\pm \mathrm{SD})$ & $87.41 \pm 6.94$ & $92.50 \pm 4.74$ & $<0.05$ \\
\hline $\mathrm{SpO}_{2}$ time $<90 \%[\%]$ (mean $\pm \mathrm{SD}$ ) & $48.63 \pm 32.26$ & $20.54 \pm 32.41$ & $<0.01$ \\
\hline Oxygen desaturation index (mean \pm SD) & $44.51 \pm 26.33$ & $5.25 \pm 5.96$ & $<0.001$ \\
\hline Apnea index (mean \pm SD) & $13.75 \pm 15.81$ & $5.25 \pm 5.96$ & $<0.001$ \\
\hline Hypopnea index (mean \pm SD) & $17.66 \pm 13.90$ & $1.39 \pm 1.30$ & $<0.001$ \\
\hline $\mathrm{AHI}(/ \mathrm{h})($ mean $\pm \mathrm{SD}$ & $32.05 \pm 22.79$ & $1.63 \pm 1.64$ & $<0.001$ \\
\hline Snore index (mean \pm SD) & $158.51 \pm 172.41$ & $128.24 \pm 151.15$ & $>0.05$ \\
\hline Arrhythmia index (mean $\pm \mathrm{SD}$ ) & $66.12 \pm 129.02$ & $0.41 \pm 0.49$ & $<0.001$ \\
\hline \multicolumn{4}{|l|}{ Neck ultrasonography data } \\
\hline $\mathrm{RPD}[\mathrm{cm}]($ mean $\pm \mathrm{SD})$ & $1.61 \pm 0.82$ & $2.32 \pm 0.28$ & $<0.05$ \\
\hline $\mathrm{DLA}[\mathrm{cm}]($ mean $\pm \mathrm{SD})$ & $2.63 \pm 0.47$ & $2.07 \pm 0.48$ & $<0.01$ \\
\hline CTBT $[\mathrm{cm}]($ mean $\pm \mathrm{SD})$ & $6.56 \pm 0.67$ & $6.72 \pm 0.77$ & $>0.05$ \\
\hline $\mathrm{STBT}[\mathrm{cm}]($ mean $\pm \mathrm{SD})$ & $7.26 \pm 0.73$ & $7.11 \pm 0.70$ & $>0.05$ \\
\hline LPWT [cm] mean \pm SD & $4.59 \pm 1.01$ & $3.41 \pm 0.71$ & $<0.001$ \\
\hline
\end{tabular}

CTBT — coronal mid-tongue base thickness; DLA — distance between lingual arteries; LPWT — lateral parapharyngeal wall thickness; N — number; Resp-LMs — respiratory-related leg movements; RPD — retropalatal pharynx transverse diameter;SD — standard deviation; STBT — sagittal mid-tongue base thickness. P value $\leq 0.05$ is significant

$(\mathrm{r}=0.542, \mathrm{P}<0.001)$ and arrhythmia index $(\mathrm{r}$ $=0.354, \mathrm{P}<0.05)$. DLA is negatively correlated with minimal oxygen saturation $(\mathrm{r}=-0.501, \mathrm{P}<$ 0.001 ) but positively correlated with $\mathrm{SpO}_{2}$ time $<$ $90 \%(\mathrm{r}=0.402, \mathrm{P}<0.01)$, oxygen desaturation index $(\mathrm{r}=0.368, \mathrm{P}<0.05)$, Apnea index $(\mathrm{r}=$ $0.317, \mathrm{P}<0.05)$ and AHI $(\mathrm{r}=0.328, \mathrm{P}<0.05)$. Also, there was a significant positive correlation between Resp-LMs index and LPWT $(\mathrm{r}=0.326, \mathrm{P}$ $<0.05)$, DLA $(\mathrm{r}=0.353, \mathrm{P}<0.05)$ and a negative correlation with RPD ( $\mathrm{r}=-0.320, \mathrm{P}<0.05)$.

From receiver operating characteristic (ROC) curve for prediction of OSA using neck ultrasonography parameters, a cutoff value of $3.47 \mathrm{~cm}$ for LPWT showed sensitivity of $100 \%$ and specific- ity of $60 \%$, and the area under the curve (AUC) was 0.855 , with high statistical significance (P $<0.001$ ), and it was the most predictive for the presence of OSA, followed by DLA with a cutoff value of $2.105 \mathrm{~cm}$ (AUC $=0.785$, sensitivity: 90.9\%, specificity: $60 \%, \mathrm{P}<0.01$ ), then RPD with a cutoff value of $1.65 \mathrm{~cm}$ as the least predictive diagnostic parameter of OSA (AUC $=0.750$, sensitivity: $54.5 \%$, specificity: $100 \%, \mathrm{P}<0.05$ ) (Figure 2). So, the combination of LPWT and RPD results can achieve a 100\% sensitivity and a 100\% specificity. Also, LPWT was found to be useful in predicting OSA severity as there was a statistically significant difference between its value in mild and moderate OSA cases (mean \pm SD $=$ 
Table 2. Collective comparison of $P$ value between neck ultrasound parameters in the four study groups

\begin{tabular}{lcccccc}
\hline & $\begin{array}{c}\text { Normal } \\
\text { vs mild 0SA }\end{array}$ & $\begin{array}{c}\text { Normal vs } \\
\text { moderate 0SA }\end{array}$ & $\begin{array}{c}\text { Normal vs } \\
\text { severe 0SA }\end{array}$ & $\begin{array}{c}\text { Mild OSA vs } \\
\text { moderate 0SA }\end{array}$ & $\begin{array}{c}\text { Mild OSA vs } \\
\text { severe 0SA }\end{array}$ & $\begin{array}{c}\text { Moderate 0SA } \\
\text { vs severe 0SA }\end{array}$ \\
\hline RPD & $\mathbf{0 . 0 0 4}$ & 0.123 & 0.107 & 0.219 & 0.148 & 1.000 \\
DLA & 0.095 & $\mathbf{0 . 0 0 6}$ & $\mathbf{0 . 0 3 6}$ & 0.247 & 0.554 & 0.347 \\
CTBT & 0.968 & 0.674 & 0.974 & 0.917 & 0.972 & 0.630 \\
STBT & 0.497 & 0.314 & 0.628 & 0.651 & 1.000 & 0.977 \\
LPWT & 0.065 & $\mathbf{0 . 0 0 2}$ & $\mathbf{0 . 0 0 1}$ & $\mathbf{0 . 0 3 4}$ & $\mathbf{0 . 0 1 8}$ & 0.514
\end{tabular}

RPD — retropalatal pharynx transverse diameter; DLA — distance between lingual arteries; CTBT — coronal mid-tongue base thickness; STBT — sagittal mid-tongue base thickness; LPWT — lateral parapharyngeal wall thickness. P value $\leq 0.05$ is significant

$3.99 \pm 0.39$ vs $5.08 \pm 1.43 ; \mathrm{P}<0.05)$ and between mild and severe OSA cases (mean $\pm \mathrm{SD}=3.99 \pm$ 0.39 vs $4.55 \pm 0.50 ; \mathrm{P}<0.05)$. LPWT was also significantly lower in the normal controls (mean $\pm \mathrm{SD}=3.41 \pm 0.71$ ) than moderate and severe OSA patients $(\mathrm{P}<0.01$ and 0.001 respectively). Also, DLA was significantly lower in normal controls (mean $\pm \mathrm{SD}=2.07 \pm 0.48$ ) than moderate $($ mean $\pm \mathrm{SD}=2.75 \pm 0.50)$ and severe $($ mean \pm $\mathrm{SD}=2.60 \pm 0.46)$ OSA cases $(\mathrm{P}<0.01$ and 0.05 , respectively). In addition, RPD was significantly lower in mild OSA cases than normal controls $($ mean $\pm \mathrm{SD}=29.43 \pm 17.13$ vs mean $\pm \mathrm{SD}=$ $34.61 \pm 14.11 ; \mathrm{P}<0.001)$. Collective comparison of $\mathrm{P}$ value between neck ultrasound parameters in the four study subgroups was shown in Table 2 .

\section{Discussion}

Obstructive sleep apnea is considered the most severe form of sleep-related breathing disorders. It can result in cardiovascular diseases, metabolic dysregulation, and neuro-cognitive dysfunction. Early diagnosis and intervention with continuous positive airway pressure (CPAP) can effectively improve airway patency, daytime wakefulness, blood pressure, metabolic abnormalities, and the quality of life [7]. Sub-mental US can detect both the anatomic characteristics, the dynamic changes of pharyngeal airspace and discriminate OSA severity [4]. The study investigated the role of ultrasonography in detecting anatomic upper airway changes in OSA patients and was trying to assess the value of these anatomical findings in prediction of OSA.

The mean age of the studied OSA patients was significantly higher than the controls'. This was consistent with Waterman et al. [8] who found that age is one of the most significant risk factors for OSA with mean \pm SD value of $67.87 \pm$
5.92 years, as age is a well-known risk factor associated with changes in the upper airway tone, pharyngeal fat distribution and the development of comorbid diseases. La Grotte et al. [9], Chen et al. [10], Jehan et al. [11] and Gray et al. [12] have shown a correlation between the prevalence of OSA syndrome and obesity (with BMI of 25$29.9 \mathrm{~kg} / \mathrm{m}^{2}, 25-29 \mathrm{~kg} / \mathrm{m}^{2}, 28-31 \mathrm{~kg} / \mathrm{m}^{2}$ and more than $30 \mathrm{~kg} / \mathrm{m}^{2}$, respectively). In a longitudinal analysis of a subset $(n=690)$ of the Wisconsin cohort with a 4-year follow-up, Peppard et al. [13] have shown that a $10 \%$ increase in weight is associated with a 6-fold greater risk of developing OSA among persons initially free of OSA. These findings were in harmony with our results.

The neck circumference of the study patients ranged from 38 to $49 \mathrm{~cm}$ with a mean \pm SD of $43.24 \pm 2.69 \mathrm{~cm}(17 \pm 1$ inches $)$. The current study agreed with several studies done by $\mathrm{Me}$ deiros et al [14] and Simpson et al. [15] who found that a large neck circumference was predictive of OSA in the general population, and it is considered a significant marker for central obesity and has been associated not only with OSA but also with increased cardiovascular risk and insulin levels in OSA patients. Neck circumference cut-points predicting OSA risk are $\geq 17$ inches $(43.2 \mathrm{~cm})$ and 16 inches $(40.6 \mathrm{~cm})$ for males and females, respectively. This study agreed with Christensen et al. [16], who evaluated the validity of ESS in diagnosing SDB and found a significant positive correlation between ESS and AHI. They concluded that if ESS $>10$, SDB should be suspected. Sleep apnea increases the risk of hypertension, cardiovascular disease, and cerebrovascular disease [17]. In agreement with our findings, Shah et al. [18] and Garcia-Rio et al. [19] reported that mild-severe OSA is an independent risk factor for MI and a risk of recurrent MI and revascularization was lower in OSA patients who 

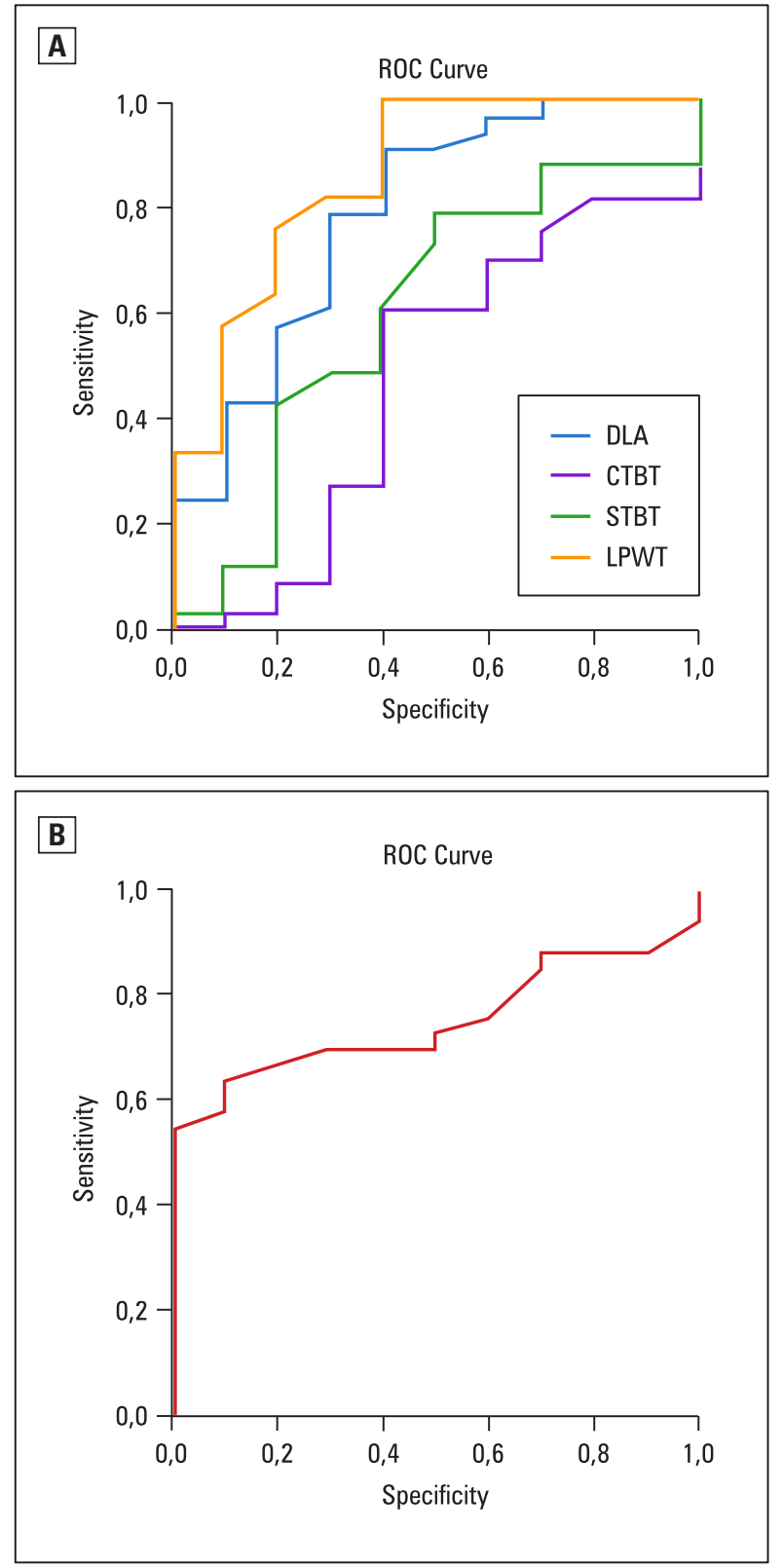

Figure 2. A. Receiver operating characteristics (ROC) curve for prediction of obstructive sleep apnea (OSA) using neck ultrasound parameters. B. ROC curve for prediction of OSA using retropalatal pharynx diameter. DLA — distance between lingual arteries; CTBT — coronal mid-tongue base thickness; STBT — sagittal mid-tongue base thickness; LPWT — lateral parapharyngeal wall thickness

tolerated CPAP. This study revealed a statistically significant difference between OSA patients and controls regarding EDS $(\mathrm{P}<0.001)$, nocturia $(\mathrm{P}<$ 0.001), snoring $(\mathrm{P}<0.01)$ and morning headache $(\mathrm{P}<0.05)$. This was consistent with Xie et al. [20] who reported that EDS is a chief clinical consequence among patients with SDB and is directly related to the severity of sleep apnea.

Regarding the comparison between OSA patients and controls in polysomnography and neck ultrasonography data, our results were consistent with Bidarian-Moniri et al. [21], who found altered AHI depending on the position and were in accordance with Yousef and Alkhiary [22] who reported significantly higher oxygen desaturation index and significantly lower minimal oxygen saturation in OSA patients than controls. As expected, AHI demonstrated significantly higher values in OSA patients than controls in this study (mean $\pm \mathrm{SD}=32.05 \pm 22.79 ;$ vs mean $\pm \mathrm{SD}=1.63 \pm$ 1.64; $\mathrm{P}<0.001$ ). Spicuzza et al. [23] mentioned that snoring, male gender, middle age, menopause in women, obesity and a variety of craniofacial and oropharyngeal features such as a large neck circumference, retro- or micrognazia, nasal obstruction, enlarged tonsils/adenoids, macroglossia and low-lying soft palate are all important factors in pathogenesis of OSA which could explain a higher AHI among these groups. The prevalence of arrhythmia in OSA patients was significantly higher than in controls (arrythmia index: mean \pm $\mathrm{SD}=66.12 \pm 129.02$ vs mean $\pm \mathrm{SD}=0.41 \pm 0.49$; $\mathrm{P}<0.001)$. Hypoxemia, reoxygenation, hypercapnia, negative intrathoracic pressure, arousal, sleep deprivation and hypertension that are associated with OSA affect electrical stability and increase the risk of arrhythmias. Likewise, Almeneessier et al. [24] found that arrhythmia in OSA patients was higher than in controls $(26.9 \%$ vs $11.5 \%$; P < 0.001) with higher incidence of premature atrial contraction, premature ventricular contraction (PVC) and atrial fibrillation in OSA patients.

Regarding correlations between neck US measurements and polysomnography parameters, our results agreed with Shu et al. [4] and Liu et al. [25] who found that enlargement of soft tissue structures, particularly the LPW, is associated with an increased likelihood of OSA. In addition, enhancement of LPWT is the principal cause of OSA among patients presenting to sleep disorders centers. Again, Lan et al. [26] reported that increased LPWT was the main cause of airway collapse, and this was significantly associated with higher oxygen desaturation index, higher AHI, higher apnea index, lower minimal $\mathrm{SaO}_{2}$, and higher $\mathrm{SpO}_{2}$ time $<90 \%$. This study revealed a highly significant positive correlation between LPWT and $\mathrm{A}+\mathrm{H} / \mathrm{h}$ in supine position, and this was confirmed by Yalciner et al. [27] who found that supine position makes the upper airway collapsible by the tongue and mandible, which is thought to be responsible for the worsening of OSA compared with non-supine position. Also, LPWT and DLA were significantly lower among this study normal subgroup than in moderate and 

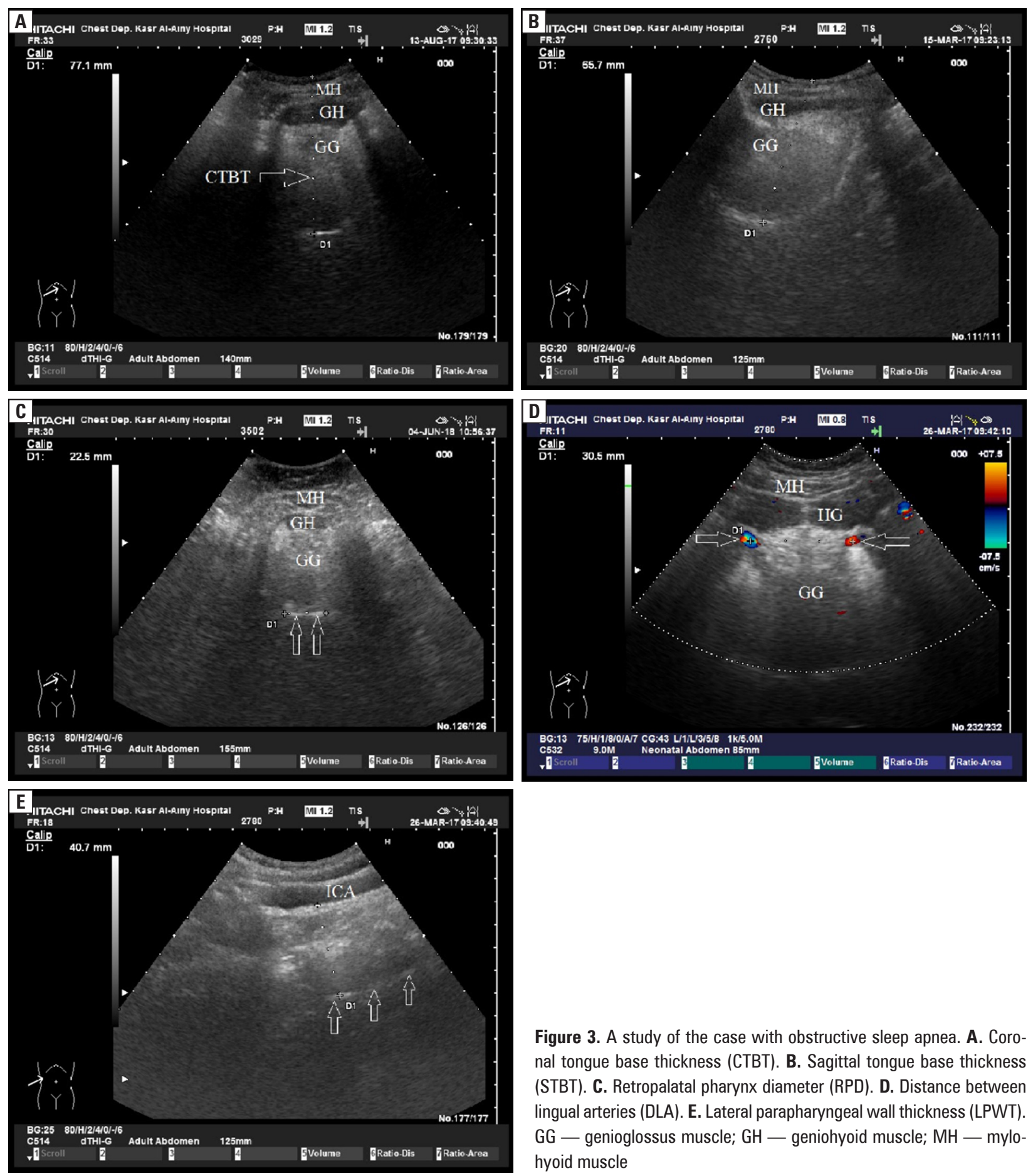

Figure 3. A study of the case with obstructive sleep apnea. A. Coronal tongue base thickness (CTBT). B. Sagittal tongue base thickness (STBT). C. Retropalatal pharynx diameter (RPD). D. Distance between lingual arteries (DLA). E. Lateral parapharyngeal wall thickness (LPWT). $\mathrm{GG}$ — genioglossus muscle; $\mathrm{GH}$ — geniohyoid muscle; $\mathrm{MH}$ - mylohyoid muscle

severe OSA subgroups, and this was in agreement with Bilici et al. [7] who stated that submental US can be used to precisely measure tongue and lateral pharyngeal wall dimensions by integrating the information. The LPWT and DLA correlate with severity of OSA as well. Lahav et al. [28], Yonatan et al. [29] and Li et al. [30] concluded that, when DLA exceeded $30 \mathrm{~mm}$, the risk for OSA was significantly increased and reported that, with cutoff point of $30 \mathrm{~mm}$ for DLA, the sensitivity, specificity, and accuracy for screening and diagnosis of OSA were 71.3, 74.7, and $63.6 \%$, respectively, which was very close to our results. This study demonstrated that DLA is negatively correlated with minimal oxygen saturation and positively correlated with $\mathrm{SpO}_{2}$ time $<90 \%$, oxygen desaturation index, AHI and apnea index. This was in agreement with a study done by Ahn et al. [31] who found that absolute tongue volume showed stronger associations with 
lowest $\mathrm{O}_{2}$ saturation during sleep than with the severity of AHI. Retropalatal pharynx transverse diameter was significantly lower in the mild OSA group than in the normal group in the present study. This finding was in agreement with Ali and Muhammad [32] who found a highly significant decrease in RPD in OSA patients compared to the controls. In addition, they observed significant progressive shortening and narrowing for RPD with progression of OSA severity and AHI. Also, Zhang et al. [33] reported that shorter retro-palatal distance was associated with higher AHI.

In the present study, STBT and CTBT were not significantly different between OSA patients and the control groups. Similar to our findings, Okubo et al. [34], Iida-Kondo et al. [35], Shigeta et al. [36] and Ahn et al. [31] found that tongue volume was not significantly different between OSA patients and the controls, and tongue volume did not correlate with BMI, AHI or RDI. Also, this study showed a significant positive correlation between Resp-LMs index and LPWT $(\mathrm{r}=0.326, \mathrm{P}<0.05)$, DLA $(\mathrm{r}=0.353, \mathrm{P}<0.05)$ and a negative correlation with RPD ( $\mathrm{r}=-0.320$, $\mathrm{P}<0.05)$. In addition, a significant positive correlation was found between STBT and isolated LMs index. This was explained by Manconi et al. [37] who mentioned that respiratory-related leg movements are provoked by respiratory-related arousals and because of the periodic nature of obstructive respiratory events, they "mimic" periodic leg movements. Thus, they are considered unique from periodic leg movements that occur as part of the phenotypic spectrum of restless legs syndrome.

The main limitation of the present study is that the cases and the control group are not matched in respect to age, BMI or neck circumference, which has definitely affected the ultrasound parameters, so we tried to divide OSA patients into grades and correlating these grades with ultrasound measurements for more accurate comparisons. In conclusion, sleep-related breathing disorders represent a spectrum of abnormalities ranging from simple snoring to upper airway resistance syndrome to sleep apnea. This study tried to highlight the role of ultrasound in OSA as a more objective tool than the questionnaire, more convenient because it does not require overnight time consumption and more relevant than pulse oximetry for examining the pharyngeal airspace. This is supported by the very good sensitivity in the present study, which demonstrates that submental US is sufficiently sensitive for differentiating OSA severity.

\section{Conflict of interest}

None declared.

\section{References:}

1. Strollo PJ, Soose RJ, Maurer JT, et al. Upper-airway stimulation for obstructive sleep apnea. N Engl J Med. 2014; 370(2): 139-149, doi: 10.1056/NEJMoa1308659, indexed in Pubmed: 24401051.

2. Khaledi-Paveh B, Khazaie H, Nasouri M, et al. Evaluation of Berlin Questionnaire Validity for Sleep Apnea Risk in Sleep Clinic Populations. Basic Clin Neurosci. 2016; 7(1): 43-48, indexed in Pubmed: 27303598.

3. Liao LJ, Cho TY, Cheng PW, et al. Submental ultrasonography in diagnosing severe obstructive sleep apnea syndrome. Journal of Medical Ultrasound. 2016; 24(3): 107-111, doi: 10.1016/j.jmu.2016.06.002.

4. Shu CC, Lee P, Lin JW, et al. The use of sub-mental ultrasonography for identifying patients with severe obstructive sleep apnea. PLoS One. 2013; 8(5): e62848, doi: 10.1371/journal. pone.0062848, indexed in Pubmed: 23675433.

5. Gay P, Weaver T, Loube D, et al. Evaluation of positive airway pressure treatment for sleep related breathing disorders in adults. Sleep. 2006; 29(3): 381-401, doi: 10.1093/ sleep/29.3.381, indexed in Pubmed: 16553025.

6. Quan SF, Budhiraja R, Batool-Anwar S, et al. Lack of impact of mild obstructive sleep apnea on sleepiness, mood and quality of life. Southwest J Pulm Crit Care. 2014; 9(1): 44-56, doi: 10.13175/swjpcc082-14, indexed in Pubmed: 25232509.

7. Bilici S, Engin A, Ozgur Y, et al. Submental ultrasonographic parameters among patients with obstructive sleep apnea. Otolaryngol Head Neck Surg. 2017; 156(3): 559-566, doi: 10.1177/0194599816684109, indexed in Pubmed: 28093961.

8. Waterman L, Stahl ST, Buysse DJ, et al. Self-reported obstructive sleep apnea is associated with nonresponse to antidepressant pharmacotherapy in late-life depression. Depress Anxiety. 2016; 33(12): 1107-1113, doi: 10.1002/da.22555, indexed in Pubmed: 27636232

9. LaGrotte C, Fernandez-Mendoza J, Calhoun SL, et al. The relative association of obstructive sleep apnea, obesity and excessive daytime sleepiness with incident depression: a longitudinal, population-based study. Int J Obes (Lond). 2016; 40(9): 13971404, doi: 10.1038/ijo.2016.87, indexed in Pubmed: 27143032.

10. Chen JW, Huang CC, Weng CK, et al. Simultaneous recording of ultrasound and polysomnography during natural sleep in patients with obstructive sleep apnea: a pilot study. J Sleep Res. 2017; 26(4): 481-486, doi: 10.1111/jsr.12515, indexed in Pubmed: 28303674 .

11. Jehan S, Zizi F, Pandi-Perumal SR, et al. Obstructive sleep apnea and obesity: implications for public health. Sleep Med Disord. 2017; 1(4), indexed in Pubmed: 29517065.

12. Gray EL, McKenzie DK, Eckert DJ. Obstructive sleep apnea without obesity is common and difficult to treat: evidence for a distinct pathophysiological phenotype. J Clin Sleep Med. 2017; 13(1): 81-88, doi: 10.5664/jcsm.6394, indexed in Pubmed: 27655455 .

13. Young T, Peppard PE, Taheri S, et al. Longitudinal study of moderate weight change and sleep-disordered breathing. JAMA. 2000; 284(23): 3015-3021, doi: 10.1001/jama.284.23.3015, indexed in Pubmed: 11122588.

14. Medeiros CA, Bruin VM, Castro-Silva Cde, et al. Neck circumference, a bedside clinical feature related to mortality of acute ischemic stroke. Rev Assoc Med Bras (1992). 2011; 57(5): 559-564, doi: 10.1590/s0104-42302011000500015, indexed in Pubmed: 22012291.

15. Simpson L, Mukherjee S, Cooper MN, et al. Sex differences in the association of regional fat distribution with the severity of obstructive sleep apnea. Sleep. 2010; 33(4): 467-474, doi: 10.1093/sleep/33.4.467, indexed in Pubmed: 20394315.

16. Christensen AS, Clark A, Salo P, et al. Symptoms of sleep disordered breathing and risk of cancer: a prospective cohort study. Sleep. 2013; 36(10): 1429-1435, doi: 10.5665/sleep.3030, indexed in Pubmed: 24082302. 
17. Jean-Louis G, Zizi F, Brown Db, et al. Obstructive sleep apnea and cardiovascular disease: evidence and underlying mechanisms. Minerva Pneumol. 2009; 48(4): 277-293, indexed in Pubmed: 21643544.

18. Shah NA, Yaggi HK, Concato J, et al. Obstructive sleep apnea as a risk factor for coronary events or cardiovascular death. Sleep Breath. 2010; 14(2): 131-136, doi: 10.1007/s11325-0090298-7, indexed in Pubmed: 19777281.

19. Garcia-Rio F, Alonso-Fernández A, Armada E, et al. CPAP effect on recurrent episodes in patients with sleep apnea and myocardial infarction. Int J Cardiol. 2013; 168(2): 1328-1335, doi: 10.1016/j.ijcard.2012.12.015, indexed in Pubmed: 23302113.

20. Xie J, Sert Kuniyoshi FH, Covassin N, et al. Excessive daytime sleepiness independently predicts increased cardiovascular risk after myocardial infarction. J Am Heart Assoc. 2018; 7(2), doi: 10.1161/JAHA.117.007221, indexed in Pubmed: 29352093.

21. Bidarian-Moniri A, Nilsson M, Attia J, et al. The effect of the prone sleeping position on obstructive sleep apnoea. Acta Otolaryngol. 2015; 135(1): 79-84, doi: 10.3109/00016489.2014.962183, indexed in Pubmed: 25384381.

22. Alkhiary AW. The severity of obstructive sleep apnea syndrome is related to red cell distribution width and hematocrit values. Journal of Sleep Disorders \& Therapy. 2015; 04(02), doi: 10.4172/2167-0277.1000192.

23. Spicuzza L, Caruso D, Di Maria G. Obstructive sleep apnoea syndrome and its management. Ther Adv Chronic Dis. 2015; 6(5): 273-285, doi: 10.1177/2040622315590318, indexed in Pubmed: 26336596.

24. Almeneessier AS, Alasousi N, Sharif MM, et al. Prevalence and predictors of arrhythmia in patients with obstructive sleep apnea. Sleep Sci. 2017; 10(4): 142-146, doi: 10.5935/19840063.20170025, indexed in Pubmed: 29410745.

25. Liu KH, Chu WCW, To KW, et al. Sonographic measurement of lateral parapharyngeal wall thickness in patients with obstructive sleep apnea. Sleep. 2007; 30(11): 1503-1508, doi: $10.1093 /$ sleep/30.11.1503, indexed in Pubmed: 18041482.

26. Lan MC, Liu SYC, Lan MY, et al. Lateral pharyngeal wall collapse associated with hypoxemia in obstructive sleep apnea. Laryngoscope. 2015; 125(10): 2408-2412, doi: 10.1002/ lary.25126, indexed in Pubmed: 25582498.

27. Yalciner G, Babademez MA, Gul F. Association of sleep time in supine position with apnea-hypopnea index as evidenced by successive polysomnography. Sleep Breath. 2017; 21(2): 289-294, doi: 10.1007/s11325-016-1401-5, indexed in Pubmed: 27572501.
28. Lahav Y, Rosenzweig E, Heyman Z, et al. Tongue base ultrasound: a diagnostic tool for predicting obstructive sleep apnea. Ann Otol Rhinol Laryngol. 2009; 118(3): 179-184, doi: 10.1177/000348940911800304, indexed in Pubmed: 19374148.

29. Lahav Y, Rosenzweig E, Heyman Z, et al. Tongue Base Ultrasound: A Diagnostic Tool for Predicting Obstructive Sleep Apnea. Annals of Otology, Rhinology \& Laryngology. 2009; 118(3): 179-184, doi: 10.1177/000348940911800304.

30. Li Y, Lin Na, Ye J, et al. Upper airway fat tissue distribution in subjects with obstructive sleep apnea and its effect on retropalatal mechanical loads. Respir Care. 2012; 57(7): 1098-1105, doi: 10.4187/respcare.00929, indexed in Pubmed: 22273428.

31. Ahn SH, Kim J, Min HJ, et al. Tongue volume influences lowest oxygen saturation but not apnea-hypopnea index in obstructive sleep apnea. PLoS One. 2015; 10(8): e0135796, doi: 10.1371/journal.pone.0135796, indexed in Pubmed: 26280546.

32. Ali E, Mohamad A. Evaluation of the efficiency of anthropometric parameters and submental ultrasonographic indices as predictors for screening of obstructive sleep apnea and its severity. Egyptian Journal of Bronchology. 2017; 11(4): 355, doi: 10.4103/ejb.ejb_17_17.

33. Zhang L, Wu Qi, Zhung Q, et al. Analysis of pharynx MRI in stroke patients with obstructive sleep apnea. Allied Journal of Medical Research. 2018; 02(01), doi: 10.35841/medical-research.2.1.1-7.

34. Okubo M, Suzuki M, Horiuchi A, et al. Morphologic analyses of mandible and upper airway soft tissue by MRI of patients with obstructive sleep apnea hypopnea syndrome. Sleep. 2006; 29(7): 909-915, doi: 10.1093/sleep/29.7.909, indexed in Pubmed: 16895258.

35. Iida-Kondo C, Yoshino N, Kurabayashi T, et al. Comparison of tongue volume/oral cavity volume ratio between obstructive sleep apnea syndrome patients and normal adults using magnetic resonance imaging. J Med Dent Sci. 2006; 53(2): 119-126, indexed in Pubmed: 16913573.

36. Shigeta Y, Ogawa T, Ando E, et al. Influence of tongue/mandible volume ratio on oropharyngeal airway in Japanese male patients with obstructive sleep apnea. Oral Surg Oral Med Oral Pathol Oral Radiol Endod. 2011; 111(2): 239-243, doi: 10.1016/j.tripleo.2010.10.013, indexed in Pubmed: 21237441.

37. Manconi M, Zavalko I, Fanfulla F, et al. An evidence-based recommendation for a new definition of respiratory-related leg movements. Sleep. 2015; 38(2): 295-304, doi: 10.5665/ sleep.4418, indexed in Pubmed: 25325500. 